\title{
Interpretation and Thinking of the "Precision Medicine Initiative" of the United States
}

\author{
Zhao Xiaoyu', Sun $\mathrm{Yu}^{2}$, Diao Tianxi ${ }^{1}$, Gao Yunhua ${ }^{1}$, Chen Ting ${ }^{1}$, Kang $\mathrm{Di}^{1}$, Wang Lei ${ }^{\text {1*}}$ and Zeng Yanjun ${ }^{3 *}$ \\ ${ }^{1}$ Institute of Health Service and Medical Information, Beijing 100850, China \\ ${ }^{2}$ Ministry of Science and Technology, Academy of Military Medical Science, Beijing, China \\ ${ }^{3}$ Beijing University of Technology, Biomedical Engineering Center, Beijing, 100022, China
}

\begin{abstract}
Background: On January 20th, 2015, President Obama proposed "Precision Medicine Initiative" in State of the Union address, which lead to close attention and rapid follow-up of many counties around the world, including China.

Methods: This paper deeply analyzes the background and motivation of the "precision medical plan" of the United States. On the basis of systematic exposition of the connotation, development history and development conditions of Precision Medicine, the advantages and disadvantages of the development of precision medicine in China are analyzed, and the thinking of the development of precision medicine in China is put forward.

Results: Five supporting conditions should be satisfied to ensure the sound development of precision medicine: To establish a new inspiring mechanism for innovations; To establish a new disease classification system incorporating molecular genetic data; To optimize the regulatory system of new drug research; Correct interpretation of genetic data; Encouraging patients to actively participate through appropriate regulations and regulatory measures.

Conclusions: During developing the precision medicine research initiative of $\mathrm{China}$, the following factors should be taken into account: focusing on prevention and control of China's major diseases; Closely combining basic medical research with clinical needs; To establish a scientific environment based on big data; To launch research on the making of relevant regulatory policies; Strengthening population-based cohort study.
\end{abstract}

Keywords: Precision medicine; Personalized medicine; Medical research

\section{Introduction}

On January $20^{\text {th }}, 2015$, President Obama proposed "Precision Medicine Initiative" in State of the Union address [1]. On January $30^{\text {th }}$, the White House issued a document to officially launch the program [2]. Obama proposed to invest $\$ 215$ million for the program in the fiscal year 2016, in order to better understand the mechanism of diseases, pave the way for the realization of "precision medicine", and "lead a new era of medicine".

\section{Profile of Precision Medicine Initiative}

\section{Core contents}

The precision medicine proposed by the United States is a new medical concept based on personalized medicine and developed with the rapid progress of genome sequencing technology and cross application of bioinformatics and big data. It carries out analysis, identification, verification and application of biological markers from a large sample of populations or specific disease types by using genomic, proteomic and advanced medical techniques. These steps enable finding the cause of disease and target of treatment, and precisely sub classifying different states and processes of a disease. Ultimately, the aim of personalized precise treatment for diseases and specific patients can be achieved, improving the diagnosis, treatment and prevention of diseases. In a nutshell, precise medicine is to create a tailored and individualized treatment regime as per the individual characteristics of each patient. Most of existing drugs are designed for "general" patients, with the undifferentiated medication. Consequently they are effective in some patients but ineffective in others. In contrast, precision medicine will help doctors better understand the complexity of a patient's condition, and thus accurately find out the most effective drug regime [3].
Francis Collins, director of the National Institutes of Health (NIH), issued a review article on Precision Medicine Initiative in the New England Journal of Medicine on March $4^{\text {th }}, 2015$. He pointed out that precision medicine is essentially personalized medicine, rather than a novel concept. Since one hundred years ago, blood typing has been used to guide transfusion scheme for patients, which is the earliest case of precision medicine. The concept of precision medicine has been put into a wider application with the fast growing of bioinformatics databases (human genome, etc.), personalized detection techniques (proteomics, metabolomics, genomics, etc.) and large data analysis technology. Compared with the traditional medical philosophy, the strength of precision medicine lies in combining the knowledge of disease mechanism with biological big data and information science, and achieving precise classification and diagnosis of diseases. Hence, the therapies provided for patients (mainly those with breast cancer or leukemia at present) will become more targeted and effective. The precision medicine features integrated biological data, targeted diagnosis and treatment of personalized medicine, and advanced realtime detection [4]. Figure 1 shows the essence of precision medicine

*Corresponding authors: Wang Lei, Institute of Health Service and Medical Information, Beijing 100850, China, Tel: 010-669-311-46; E-mail: wangleienjoy@163.com

Yanjun Zeng, Beijing University of Technology, Biomedical Engineering Center Beijing, 100022, China, Tel: 010-673-918-09; E-mail: yjzeng@bipu.edu.cn

Received March 01, 2016; Accepted April 13, 2016; Published April 18, 2016

Citation: Xiaoyu Z, Yu S, Tianxi D, Yunhua G, Ting C, et al. (2016) Interpretation and Thinking of the "Precision Medicine Initiative" of the United States. J Bioprocess Biotech 6: 277. doi:10.4172/2155-9821.1000277

Copyright: (C) 2016 Xiaoyu Z, et al. This is an open-access article distributed unde the terms of the Creative Commons Attribution License, which permits unrestricted use, distribution, and reproduction in any medium, provided the original author and source are credited. 


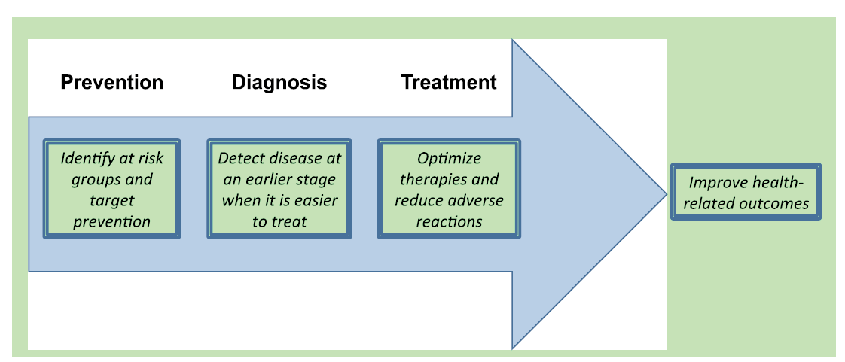

Figure 1: Expected benefits of precision medicine at different stages of clinical treatment.

at every stage of treatment, that is, to establish diagnosis, prevention and treatment method satisfying personalized needs of every patient through molecular medicine approaches.

\section{Objectives and main contents}

The short-term goal of Precision Medicine Initiative is to search more and better therapies for cancers, while its long-term goal is to provide valuable information for realizing personalized treatments for multiple diseases. According to the proposal of Obama, the program will obtain $\$ 215$ million budget in the fiscal year 2016. Therein, $\$ 130$ million will be allocated to the National Institutes of Health (NIH) to support research groups, volunteer recruitment, and establishment of biological sample library. The task of NIH is to gather data from 1 million people including their medical records, genes and lifestyles. Meanwhile, $\$ 70$ million will be used to support the National Cancer Institute (NCI) for research on formation mechanisms and drugs of cancers. Moreover, $\$ 10$ million will be offered to the U.S. Food and Drug Administration (FDA), aiming to build regulatory frameworks for project databases, and the Office of the National Coordinator for Health Information Technology (ONC) will also get $\$ 5$ million with the mission of establishment of standards to ensure that data sharing will not infringe on personal privacy.

\section{Background of Precision Medicine Initiative}

\section{The philosophy of precision medicine has a long history}

The essence of precision medicine is personalized medicine, which was first proposed in 1970s. But due to the limited level of medical science and technology at that time, it did not arouse much attention from the research and medical field. Until the Human Genome Project was completed in 2002, the term personalized medicine began to appear frequently in various medical journals, and became a promising development direction for medical research. But it remained in the infancy stage.

\section{National think tank plays an important role in the policy making for sciences and technologies}

In 2011, Dr. Maynard V. Olson, a famous scholar of genome sciences, participated in the drafting of a report "Towards Precision Medicine", which was jointly issued by the U.S. national think tanks. The report officially put forward the concept of precise medicine, that is, "to achieve effective warning and accurate treatment of human diseases by means of closely linking genetics-related researches to the clinical medicine". Twenty five years ago, he was also involved in drafting another epoch-making report of think tanks, "Human Genome
Sequencing". Precision Medicine Initiative is launched on the basis of continuous research on technology roadmaps and strategies carried out by the U.S. national think tanks, which reflects the important influence of think tanks on the U.S. Government [5].

\section{Driven by political, economic and scientific factors}

By analysis of the background of Precision Medicine Initiative announced by Obama, three driving factors were being concluded. The first is the political factor. In recent years, the drastically increased medical expenses, waste of medical resources and excessive medical treatment land the health care reform of the U.S. in a predicament. Thus Obama urgently needs to take measures to reverse "the failure of health care reform" which would cause adverse effects to public health. Precision Medicine Initiative is timely introduced in such a background, demonstrating high concerns of the U.S. government to the public health.

Economy is the second factor. This program will invigorate the economy. Its implementation is largely dependent on the advancement of next-generation sequencing. At present, the global market of next-generation sequencing is estimated to be $\$ 20$ billion. And this technique will greatly promote drug discovery, cancer diagnostics and personalized clinical application. The market of next-generation sequencing develops so rapidly that the future scale is difficult to estimate.

The third factor is science and technology. Biotechnology, especially the rapid progress of gene sequencing technology, has paved the way to precision medicine. Recently, genetic diagnosis and therapy has made a great breakthrough. Personalized diagnosis is carried out on the basis of gene sequencing with the aim of connecting DNA sequences with diseases or signs. On the one hand, DNA sequencing is required to be faster, more accurate and low-cost; on the other hand correlation is needed to be found between biological information and diseases. Currently the most advanced sequencing instrument has achieved high throughput, so that a whole genome can be sequenced rapidly. Furthermore, the cost has dropped to $\$ 1000$, making wide clinical applications possible. Groundbreaking progress has been made in association study between various genetic mutations and diseases. It has been confirmed that many human diseases are directly or indirectly linked to genes.

\section{Results and Discussion}

\section{Prerequisites for development of precision medicine}

In October 2012, the World Economic Forum released a report named Preparing for Precision Medicine [6]. The report pointed out that precision medicine is the development direction of the future medical science, but its development environment has not yet been fully mature; five supporting conditions should be satisfied to ensure the sound development of precision medicine.

\section{To establish a new inspiring mechanism for innovations}

The central idea of precision medicine is to ensure every patient to get a suitable treatment in the right time, which will undoubtedly bring significant social benefits. But it also means a new treatment mode, i.e., a drug can only be provided to a small number of patients. This idea has challenged the traditional pharmaceutical business model based on the "blockbuster drug". Owning to massive capital investments required in new drug research and development, pharmaceutical companies tend to develop a drug suitable for more people. Comparatively, 
they less enthusiastic about targeted drugs that apply to only a small population but are high-cost and terribly uncertain in return. Therefore, appropriate incentives and reimbursement schemes need to be established to encourage pharmaceutical companies to develop targeted drugs in accordance with the concept of precision medicine.

\section{To establish a new disease classification system incorporating molecular genetic data}

The rapid development of human genome sequencing and high throughput technology has greatly enhanced the understanding of diseases. It is recognized that the shift from healthy to unhealthy state involves a large number of activities at the molecular level. Therapeutics of a disease in accordance with the precision medicine cannot be established until the disease is understood at the level of molecular biology. This inevitably requires the establishment of a new disease classification system that incorporates the emerging molecular medicine knowledge. The current international classification of disease standards established by World Health Organization cannot provide enough information about the disease-associated molecular medicine information. This defect separates the research results of molecular medicine from clinical diagnosis and treatment. The new disease classification system designed for precision medicine will greatly integrate molecular medicine and clinical medical data.

\section{To optimize the regulatory system of new drug research}

The sound development of precision medicine requires fast discovery and development of a new generation of drug therapy. At present, a lot of medical research organizations are appealing to regulators for improving the new-drug regulatory process. Lengthy approval process is considered to be a great obstacle to the effective transformation from laboratory result to clinical applications. The research result of Kesselheim et al. [7] also confirmed this view. The number of new drugs approved by FDA decreased by $39 \%$ in 2005 2009 than in 1995 1999, while investments in the field of biomedical research rose sharply. The article indicated that the decline of approved drug number is fundamentally due to the uncertainty and irrationality of identification criteria for safety and efficacy of new drugs in the approval system, which is not conducive to the development of innovative drugs. Therefore, an efficient new-drug regulatory system is called for by the scientific community to facilitate the development of precision medicine. The new regulatory process should discard regulatory procedures for traditional clinical trials. More attention should be paid to selecting specific patients in phase III clinical trials, in order to optimize the process and improve the economic and clinical outcomes.

\section{Correct interpretation of genetic data}

Precision medicine needs to deal with a large number of clinical data, and interpret numerous basic omics data. At present, most clinicians cannot explain the relationship between the result of molecular biology test and the risk of disease. A few countries have carried out trainings on genetic information analysis, in which clinicians are specially trained to explain genetic test results to patients.

\section{Encouraging patients to actively participate through} appropriate regulations and regulatory measures

As the research method of precision medicine will lead to a series of ethical issues: whether the use of invasive treatments is appropriate for the healthy population at risk? Will the use of biological information increase the inequality between patients? What kind of privacy individuals enjoy on their own biological information, and how to protect their information safety? Who has the right to use the genetic and biological information? Who has the right to "own" the genetic and biological information? Only solving these problems by improving legislative and regulatory measures, can patients be motivated to actively provide their own genetic information and participate in the study of precision medicine.

\section{Advantages and Disadvantages of China in Carrying Out the Research of Precision Medicine}

\section{Advantages of China's development of precision medicine research}

China has made considerable progresses in recent 30 years in the field of genome sequencing, molecular typing of clinical diseases, markers for diagnosis and treatment, target-based drug design, cohort study and biomedical data. Hence, China boasts following advantages in the development of precision. First, a number of research bases and teams with international competitiveness have been formed. Especially the gene sequencing capacity has stepped into the leading position of the world, which lays foundations of both personnel and technology for China to carry out the research and application of precision medicine. Second, China has an absolute advantage in size of cohort study on healthy and unhealthy population, as well as diversity of biological samples. The third advantage lies in the speed and cost of large-scale, multicenter clinical trials. The fourth advantage is embodied in the flexibility of data collection and use.

\section{Weakness in precision medicine research}

There are still several constraints for China to carry out research on precision medicine. First, core sequencing instruments and key frontier technologies needed to carry out the research of precision medicine are heavily dependent on import. China's capacities of independent research and development and innovations have certain gaps from the international level. Second, there is a lack of sharing mechanism between medical database and biological sample libraries. Accordingly, the "information island" phenomenon is very common, and obstacles are faced in data exchange, integration and use. Third, translational medicine has great potential to improve. The basic medical research results in disease monitoring, diagnosis and treatment are urgently needed to be translated into clinical practices. Fourth, the policies and legal system for supervising genetic diagnosis, security of patient health information, and new clinical technologies and products [8].

\section{Conclusions}

\section{Thinking and enlightenment}

The Precision Medicine Initiative proposed by the U.S. is a new medical research plan with "holistic thinking", conforming to the times and meeting requirements of science and technology development. This program is derived from "personalized medicine" and systematically integrates its research techniques and methods (genomics, proteomics, metabolomics, pharmacogenomics, etc.) as well as research resources (cohort data of healthy and unhealthy population, biological sample libraries and electronic health records). A grand precision medicine plan of the U.S. medical community is thus outlined, characterized by the systematic integration of medical research. It is suggested that the "precision medicine research initiative of China" should be implemented to meet needs of scientific and technological development. Before the implementation of this program, we should learn from the experiences 
of Precision Medicine Initiative in the U.S., such as the holistic thinking of integration of medical research systems, top-level design, overall layout, and foresight in dealing with the relationship between precision medicine and other biomedical research, which promotes the coordinated development of national pharmaceutical science and technology. During developing the precision medicine research initiative of China, the following factors should be taken into account.

\section{Focusing on prevention and control of China's major diseases}

The Precision Medicine Initiative of the U.S. is mainly aimed at cancers, which should not be blindly copied. We should be devoted to control and prevent those major diseases in China, creating our own mode, path and guidelines of precision medicine research. At the same time, it is expected that our country possibly takes the lead in the field of personalized disease prevention, if we incorporate he research idea and methods of precision medicine into the "preventive treatment of disease" theory of traditional Chinese medicine to study the precision and personalized disease prevention.

\section{Closely combining basic medical research with clinical needs}

The Precision Medicine Initiative of the U.S. reminds us that the scientific research in basic medicine, especially in genomics, proteomics, metabolomics, etc. should not be carried out alone. The advantages of basic medical research and clinical studies should be integrated, and the information sharing and mining between clinical samples and clinical data should be attached great attention. Thus, it will become easier to promote personalized and precision study on etiology, and search for accurate therapeutic targets and drugs.

\section{To establish a scientific environment based on big data}

Electronic medical records established by most clinical hospitals in China only solve the problem of paperless office, but cannot provide structured data. Hence, there is a lack of big data that can be shared and utilized by precision medicine research. It is urgent to establish a unified, standard and structured electronic clinical record and a data sharing system with unified encoding, paving the way towards precision medicine.

\section{To launch research on the making of relevant regulatory policies}

In the Precision Medicine Initiative of the U.S., the application of science and technology and the construction of related regulation, assessment and supervision systems are underway simultaneously. It suggests that we should proactively carry out regulatory mechanism and policy researches, including those concerning the supervision of gene diagnosis, security of patient health information, and new clinical technologies and products, thus providing support for the formulation of relevant laws and regulations.

\section{Strengthening population-based cohort study}

The United States has carried out many long-term population-based cohort studies, which are the important basis for precision medicine research. For example, University of Massachusetts Boston has been committed to the "Framingham Heart Study" of NIH for 67 years, aiming to clarify risk factors for heart diseases. The "Million Veteran Program" of the U.S. Department of Veterans Affairs concentrates on studying the impact of gene on health. China should grasp new opportunities and actively launch large cohort studies of healthy and unhealthy people, providing data for the disease risk detection and personalized treatment based on precision medicine.

\section{Authors' Contributions}

Zhao Xiaoyu carried out the studies on the Profile of Precision Medicine Initiative and made total design of the paper. Sun Yu carried out the research on Background of Precision Medicine Initiative and made total design of the paper. Diao Tianxi participated in the research on Prerequisites for development of precision medicine. Gao Yunhua participated the analysis of Advantages and disadvantages of China in carrying out the research of precision medicine. Chen Ting participated the analysis of Advantages and disadvantages of China in carrying out the research of precision medicine. Kang Di participated the analysis of Advantages and disadvantages of China in carrying out the research of precision medicine. Wang Lei and Zeng Yanjun conceived of the study, and participated in its design and coordination and helped to draft the manuscript.

\section{References}

1. The White House (2015) President Obama's State of The Union AddressRemarks as Prepared for Delivery.

2. Fact Sheet: President Obama's Precision Medicine Initiative.

3. Bert A (2014) AAAS President: 'The Promise of Convergence'. Elsevier.

4. Collins FS, Varmus $\mathrm{H}$ (2015) A new initiative on precision medicine. N Engl J Med 372: 793-795

5. (2011) Toward Precision Medicine: Building a Knowledge Network for Biomedical Research and a New Taxonomy of Disease. The National Academies Press.

6. (2012) Preparing for Precision Medicine. World Economic Forum

7. Kesselheim AS (2010) Using market-exclusivity incentives to promote pharmaceutical innovation. N Engl J Med 363: 1855-1862.

8. Zhang J (2015) An interview with Academician Cao Xue-tao: Promoting the development of precision medicine to make Chinese more healthier. 